\title{
Revisiting the reconstructive surgery framework: the reconstruction supermarket
}

Hari Venkatramani ${ }^{1}$, Jeremy N Rodrigues ${ }^{1,2}$, S Raja Sabapathy ${ }^{1}$

${ }^{1}$ Department of Plastic, Hand and Reconstructive Microsurgery and Burns, Ganga Hospital, 313, Mettupalayam Road, Coimbatore 641043, Tamil Nadu, India

${ }^{2}$ Nuffield Department of Orthopaedics, Rheumatology and Musculoskeletal Sciences (NDORMS), University of Oxford, Oxford, UK

Plastic and reconstructive surgeons apply a variety of techniques throughout the body with analogies having evolved to provide a conceptual framework for the selection of techniques. Well-known examples include: the reconstructive ladder with increasingly complex rungs, the reconstructive elevator where the surgeon moves directly to the most suitable reconstruction, the reconstructive toolbox, and the reconstructive triangle. ${ }^{1,2}$ Extended models have been proposed to incorporate developments such as negative pressure wound therapy, and include the extended ladder, ${ }^{3}$ the reconstructive solar system, ${ }^{4}$ and the reconstructive clockwork. ${ }^{5}$ All have advantages with individuals favouring particular models when learning or teaching. However, other factors influence reconstruction decision-making, and do not always fit within these existing analogies. These include awareness of variants of flap options, insight into one's level of experience with different flaps, and the rise of value-based health care, in which patients may have individualised requirements and interpretations. We propose an alternative framework that may help to incorporate these issues while supporting a patient-centred approach to reconstruction.

\section{The Reconstructive Supermarket}

The great variety of reconstructive options that plastic surgeons may deliver are classified in different ways. Many reconstructive algorithms focus on the technique employed, such as grafts versus flaps. Flaps themselves are subdivided into pedicled or free, or by composition 
for example. It is notable that many different flap compositions and options can be harvested as variants on a theme, or from nearby sites. Such sites include the thoracodorsal axis, the groin, the anterolateral thigh, and the medial thigh. Each of these anatomical areas can be considered analogous to an aisle within a supermarket that comprises all of them.

Surgeons visit the supermarket with the aim of putting the options needed in the shopping basket for the reconstruction. The first key principle is that we shop on behalf of the patient, who ultimately foots the bill. Good clinical care and respect for the nature of this arrangement is essential to remember. Some supermarkets are less well-stocked with fewer options available and some patients are less able to afford an expensive shopping bill. The surgeon should have insight to the context of the patient's case.

\section{Supermarket aisles and anatomical regions}

Related products tend to be located near to each other within an aisle of a supermarket. The same applies to many of the anatomical regions from which many flaps are harvested. In the anterolateral thigh, a perforator flap, a fasciocutaneous flap, a fascial flap, a myocutaneous flap, a muscle flap, a reverse flow flap, and a flow through flap can all be raised. Other "aisles", such as the groin, include graft options (skin or bone) and flap options such as the deep circumflex iliac artery flap and the groin flap. Some exemplar reconstructive supermarket "aisles" could include:

- Anterolateral thigh

- Medial thigh

- Groin

- Axilla

- Abdomen

- Neck 


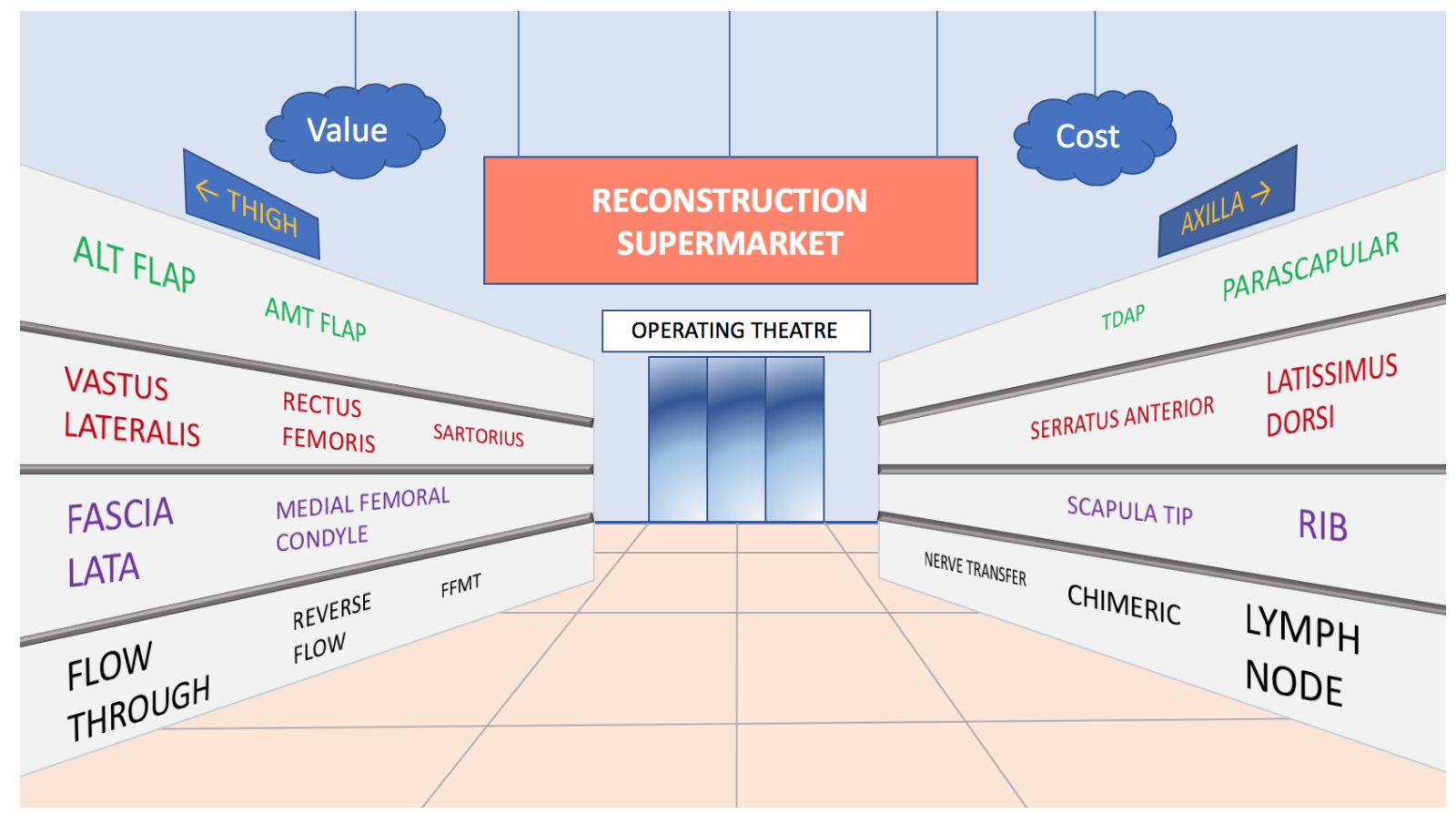

Figure 1: Conceptual schematic of the reconstruction supermarket, with some contents of two "aisles" illustrated.

ALT: anterolateral thigh, AMT: anteromedial thigh, FFMT: free functioning muscle transfer, TDAP: thoracodorsal artery perforator flap

\section{Selecting combinations of items}

When selecting items, several may be needed to meet the requirements of a defect, just like shopping to follow a recipe. These may be related products, found in the same aisle of a supermarket, such as raising a chimeric flap of serratus anterior along with latissimus dorsi. Alternatively, the different products needed may be found in different areas, requiring familiarity with different aisles. For example, sural nerve grafts and plantaris tendon grafts from the calf may be combined with a free anterolateral thigh flap to reconstruct a hand. A shopping list of what products are required can be made by considering the recipe in advance and doing so will ensure that the shopping trip is planned in a logical and efficient order. It may also avoid the difficult situation when the surgeon finds that a key ingredient is not in stock and as such unavailable for use. 


\section{Surgeon expertise and familiarity with the layout and options available}

In addition to planning the shopping required for a patient, the surgeon's familiarity with the goods available is important. Within an aisle, if one always selects the same product and does not periodically review the other options available on nearby shelves, then those other options may not come to mind when they would be the best option for a patient. Similarly, if a surgeon never visits a particular aisle, then he or she will not be comfortable and efficient in obtaining products located in that aisle. The need for continuing education follows this pattern too - even if a surgeon was familiar with an aisle in the past, the range of products there may be updated from time to time with new developments. Lifelong learning may minimise this risk.

\section{Goals, reviews and feedback}

Nowadays, products in a supermarket may be accompanied by reviews from previous purchasers. Such feedback can be useful for making informed choices. Feedback from patients may lead to a surgeon changing his or her mind about product selection, leading to more satisfied consumers. Mechanisms to achieve this feedback need to be supported, and these include direct feedback from patients, initiatives such as national registries of procedures, and awareness of contemporary scientific literature in the field. It should be borne in mind that the opinions in reviews can vary between individuals. Different interpretations and experiences can lead to people recommending alternative products. The preference for different supermarket products or brands is analogous to ongoing differences in opinion in reconstructive surgery. The debate between the use of muscle flaps and fasciocutaneous flaps for open lower limb trauma cover exemplifies this. Different individuals may advocate one or other, and there are merits to both. ${ }^{6}$ Preferences may reflect different personal experiences with reconstructive options, different training, or a different patient catchment. Just as different products from a supermarket can be used in a recipe, so different reconstructive solutions may be provided by different surgeons and achieve comparable results. There is not always a single best option. 
Furthermore, just as consumer reviews of products may differ from what might be 'objectively' expected by experts in field, so patient opinions and preferences about reconstruction may be based on criteria that surgeons may not anticipate.

In keeping with the principles of value-based health care, reconstructive options chosen should align with the individual patient's circumstances and requirements.

\section{Value}

This conceptual framework supports higher order thinking approaches when appraising reconstructive options, by incorporating a range of factors that influence decision-making. In addition to the points discussed, a final aspect of this analogy is the need for value. Just as the supermarket will strive to deliver value for money to consumers, so the reconstructive surgeon must ensure that the options that they deliver to patients deliver value for them.

\section{References}

${ }^{1}$ Gottlieb LJ, Krieger LM. From the reconstructive ladder to the reconstructive elevator. Plast Reconstr Surg 1994; 93(7): 1503

${ }^{2}$ Mathes S, Nahai F. Reconstructive Surgery: Principles, Anatomy \& Technique. New York: Churchill-Livingstone. 1997

${ }^{3}$ Janis JE, Kwon RK, Attinger CE. The New Reconstructive Ladder: Modifications to the Traditional Model. Plast Reconstr Surg 2011; 127 (suppl.): 205S

${ }^{4}$ Giordano V, Napoli S, Quercioli F, Mori A, Dini M. The Solar System Model for the Reconstructive Ladder. Plast Reconstr Surg 2011; 128(1): 336-7

${ }^{5}$ Knobloch K, Vogt PM. The reconstructive clockwork of the twenty-first century: An extension of the concept of the re- constructive ladder and reconstructive elevator. Plast Reconstr Surg $2010 ; 126: 220 e-222 e$

${ }^{6}$ Cho EH, Shammas RL, Carney MJ, Weissler JM, Bauder AR, Glener AD, Kovach SJ, Hollenbeck ST, Levin LS. Muscle versus Fasciocutaneous Free Flaps in Lower Extremity 
Traumatic Reconstruction: A Multicenter Outcomes Analysis. Plast Reconstr Surg 2018 141: $191-9$

\section{$\underline{\text { Conflicts of interest }}$}

JNR is funded by the NIHR. The views expressed are those of the authors, and are not necessarily those of the NIHR, the NHS or the Department of Health. 